\title{
ERROR ANALYSIS FOR THE AIRBORNE DIRECT GEOREFERINCING TECHNIQUE
}

\author{
Ahmed S. Elsharkawy ${ }^{\mathrm{a} *}$. Ayman F Habib ${ }^{\mathrm{b}}$ \\ ${ }^{a}$ MTC, Civil Engineering Department'Cairo, Egypt - ahmadshawky007@yahoo.com \\ ${ }^{\mathrm{b}}$ Professor, Lyles School of Civil Engineering, Purdue University, USA - ahabib@ purdue.edu
}

Commission I, WG I/3

KEY WORDS: Direct Georeferencing, error analysis, EOP, IOP, GCPs, ISO and GPS supported AT.

\begin{abstract}
:
Direct Georeferencing was shown to be an important alternative to standard indirect image orientation using classical or GPS-supported aerial triangulation. Since direct Georeferencing without ground control relies on an extrapolation process only, particular focus has to be laid on the overall system calibration procedure. The accuracy performance of integrated GPS/inertial systems for direct Georeferencing in airborne photogrammetric environments has been tested extensively in the last years. In this approach, the limiting factor is a correct overall system calibration including the GPS/inertial component as well as the imaging sensor itself. Therefore remaining errors in the system calibration will significantly decrease the quality of object point determination.

This research paper presents an error analysis for the airborne direct Georeferencing technique, where integrated GPS/IMU positioning and navigation systems are used, in conjunction with aerial cameras for airborne mapping compared with GPS/INS supported AT through the implementation of certain amount of error on the EOP and Boresight parameters and study the effect of these errors on the final ground coordinates.

The data set is a block of images consists of 32 images distributed over six flight lines, the interior orientation parameters, IOP, are known through careful camera calibration procedure, also 37 ground control points are known through terrestrial surveying procedure. The exact location of camera station at time of exposure, exterior orientation parameters, EOP, is known through GPS/INS integration process. The preliminary results show that firstly, the DG and GPS-supported AT have similar accuracy and comparing with the conventional aerial photography method, the two technologies reduces the dependence on ground control (used only for quality control purposes). Secondly, In the DG Correcting overall system calibration including the GPS/inertial component as well as the imaging sensor itself is the limiting factor to achieve good object space.
\end{abstract}

\section{INTRODUCTION}

The determination of the exterior orientation parameters (e.g. position $\mathrm{X}, \mathrm{Y}, \mathrm{Z}$ and attitude $\omega,=\phi,=\kappa$ of an image at the time of exposure) is an essential pre-requisite for the evaluation of imagery based on any type of data from terrestrial, airborne or satellite platforms.

Traditionally, in photogrammetry this orientation task is solved indirectly using the well known method of aerial triangulation (AT). Although aerial triangulation was essentially improved and expanded to so called automated aerial triangulation (AAT) or Integrated Sensor Orientation (ISO) in the last years (e.g. Schenk (1997)), the orientation process still suffers from a large amount of interactive editing and supervision of highly skilled operators. This is especially due to the high computational effort that is necessary for automatic tie point measurement. A reliable matching of tie points is necessary to determine the exterior orientation of each image correctly, with the availability of integrated GPS/inertial systems this situation changes, GPS offers high absolute accuracy position and velocity information. The short term noise is dependent on the data quality and observation approach. In contrast to this, inertial systems provide very high relative accuracy for position, velocity and attitude information, but the absolute accuracy decreases dependent on runtime if the system is working in stand-alone

*Ahmed Shawky Elsharkawy, Lecturer in the Civil Engineering Department, Military Technical College, Cairo, Egypt 
mode and no external update measurements are available.

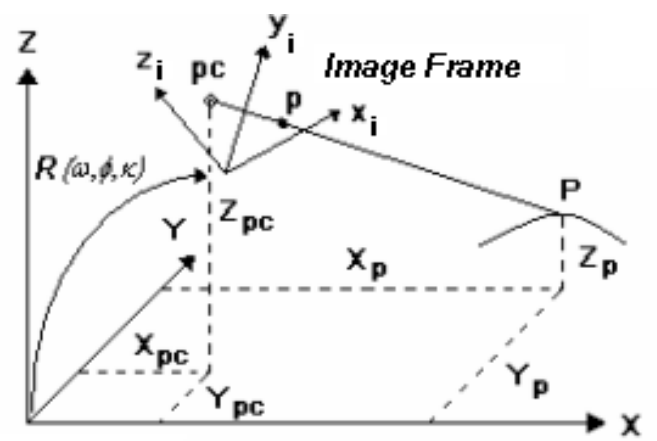

\section{Mapping Frame}

Figure 1. Exterior Orientation Parameter

Since GPS and inertial systems are of complementary error behaviour, their optimal integration allows fully exterior orientation determination with improved overall accuracy and at higher reliability compared to the stand-alone units. Meanwhile integrated GPS/inertial systems are commercially available and commonly used for the operational processing of digital airborne sensor data.

Within the following section the Principles of Indirect and Direct Georeferencing are described. The Methodology used and the result of a test flight performed over the area of British Columbia is discussed in section 3 . Then the performance criterion is described in section 4. Comments on the results and analysis are introduced in section 5 . Finally conclusions are summarized in section 6.

\section{LITERATURE REVIEW}

\subsection{Indirect Georeferencing}

Aerial photogrammetry, has been widely used for high accuracy mapping applications at all scales. Actually, all operation methods was relying on the existence of Ground Control Points (GCPs) as they were the mandatory supply of information for providing the Georeferencing parameters and eliminating unwanted error propagation. This fact extended even with the main changes in photogrammetry from analogue to analytical and then to the digital mode of operation, it was taken for granted that GCPs were the only source for providing reliable Georeferencing information. In order to minimize the number of GCPs, external Georeferencing information was therefore used (Habib, Ghanma et al. 2005). The drawback of indirect Georeferencing is the cost associated with the establishment of the GCPs. They usually represent a significant portion of the overall budget. In some cases, this cost can be unaffordable; especially when imagery is to be acquired and georeferenced in remote areas such as areas found in many developing countries (El-Sheimy 2008). The use of the GCPs also often puts operational limitations on a specific flight mission.

The use of additional position and navigation sensor data in the Georeferencing process has been extensively studied for some decades. The output of these sensors is used to determine the six parameters of exterior orientation, either completely or partially, and thus to eliminate the need for a dense GCP network. However, at this stage the use of the supplementary data was intended only to support the Georeferencing process by reducing the number of GCPs. Also, the accuracy achieved with most of these auxiliary data was limited. Thus, during the last two decades the use of such auxiliary data in the Georeferencing process has almost disappeared completely from photogrammetry (Ackermann 1995)

This situation changed fundamentally when GPS data were included in the block adjustment of aerial triangulation. In principle, the use of GPS data made block triangulation entirely independent of GCPs. Therefore, the Georeferencing process became autonomous, as GCPs were not necessarily required any more. However, this is only true for the block triangulation scheme with over-lapping images. Other sensors cannot be fully georeferenced by GPS alone. Well-known examples of which are pushbroom digital scanners, laser scanners, and imaging radar systems, which are important in kinematic mapping applications (El-sheimy 2008).

\subsection{Direct Georeferencing}

Multi-sensor systems have become an emerging trend in mapping applications because they allow a task-oriented implementation of geodetic concepts at the measurement level. These systems have a common feature in that the sensors necessary to solve a specific problem are mounted on a common platform. By precise synchronizing the data streams, the solution of a specific problem is possible by using data from one integrated measurement process only, and also offers in most cases the potential for real-time solution, which is becoming more important in many applications (Schwarz and ElSheimy 1996).

In CCD camera images, the following information is needed for a pair of cameras: 
- Position of the camera perspective center at exposure time (3 parameters per image),

- Camera orientation at exposure time (3 parameters per image),

- Interior geometry of the camera sensor, and

- Lens distortion parameters.

The first two sets of parameters are known as exterior orientation parameters, while the other two sets are known as interior orientation parameters. The general problem in photogrammetry, aerial and terrestrial, is the determination of the camera's interior and exterior orientation parameters. The exterior orientation parameters are determined by a combination of GPS and INS, the interior orientation parameters by field or laboratory calibration. This means that exterior orientation is tied to a real-time measurement process and its parameters change quickly. In contrast, interior orientation is obtained by using a static field calibration procedure and can be considered as more or less constant for a period of time. Thus, it can be done before or after the mission and is not generally affected by the data acquisition process. When the navigation information is provided by an integrated INS/DGPS system, the equation for direct Georeferencing takes the form:

$$
\boldsymbol{r}_{i}^{m}=\boldsymbol{r}_{i n s / d g p s}^{m}(t)+\boldsymbol{R}_{b}^{m}(t)\left[s_{i} \boldsymbol{R}_{c}^{b} \boldsymbol{r}_{i}^{c}(t)+\boldsymbol{a}^{b}\right]
$$

Where:

$$
\begin{array}{ll}
r_{i}^{m} & \begin{array}{l}
\text { is a vector of coordinates to be } \\
\text { computed in the mapping frame for a } \\
\text { specific point (i), }
\end{array} \\
r_{\text {ins/dgps }}^{m}(t) \begin{array}{l}
\text { is a vector containing the coordinates } \\
\text { of the INS center in the mapping }
\end{array} \\
\text { frame, determined by the INS/DGPS } \\
\text { integration } \\
R_{b}^{m}(t) \quad \begin{array}{l}
\text { is the attitude matrix from the INS } \\
\text { body frame to the mapping frame, } \\
\text { determined by the INS/DGPS } \\
\text { integration }
\end{array}
\end{array}
$$

$\mathrm{s}_{\mathrm{i}} \quad$ is a scale factor between the image and mapping coordinate frames for a specific point (i), usually determined by processing the captured imagery in stereo pairs

$R_{c}^{b} \quad$ is the rotation matrix (orientation offset) between the camera frame and the INS body frame determined from calibration

$r_{i}^{c}(t) \quad$ is the vector of coordinates (i.e., $\mathrm{x}, \mathrm{y},-$ f) observed in the image frame for a specific image (t) and point (i),

$r^{b} \quad$ is the vector of the translation offset between the INS and the camera centre in the INS body frame determined by terrestrial measurements as part of the calibration process(El-Sheimy 2008)

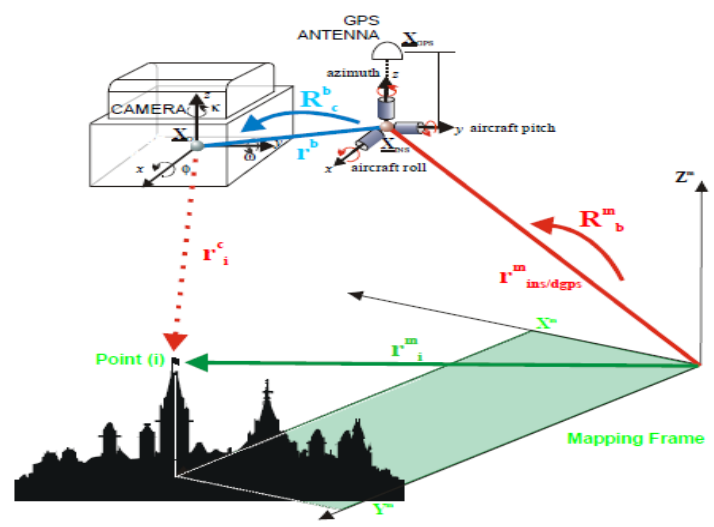

Figure 2. Elements of Direct Georeferencing

The relations between the components of Equation (1) are depicted in Figure 2. This formula expresses the fact that the processing chain contributing to the overall performance of an acquisition system is affected by the accuracy of the measured image data, the INS/DGPS position and attitude, the system calibration, the optical properties of the cameras and the effect of image geometry.

- Sources of errors in Direct Georeferencing
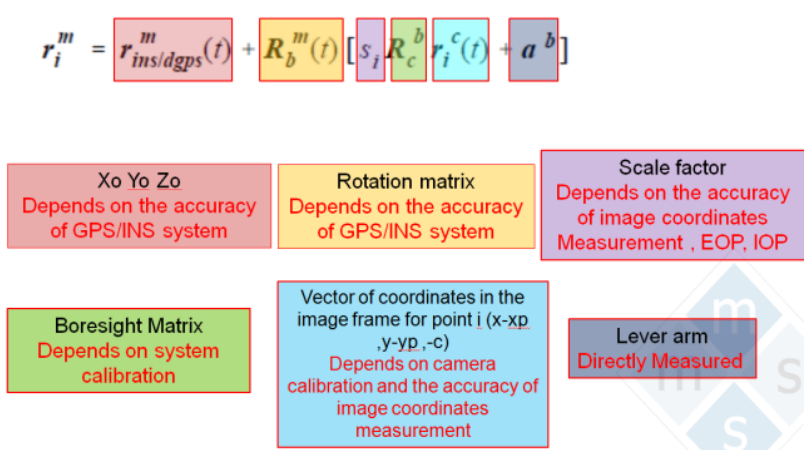

Figure 3. Sources of errors in Direct Georeferencing

As depicted in Figure 3 we have three groups of errors depending on the parameters involved in each group, the first group is the error in the shift component or the position vector of the center of the INS body frame in mapping frame (Xo Yo Zo ), the rotation matrix between the mapping frame and body frame $(\omega, \phi$ and $\mathrm{K})$ and finally the scale factor which depend on the previous factors plus the IOP parameters.

The second group of errors is the Boresight matrix and Lever arm components which depends on system calibration 
The third group of error is the vector of coordinates of point $\mathrm{i}$ in the image frame which depends on the camera calibration and the accuracy of image coordinates measurements.

\section{THE METHODOLOGY}

The data set we have is a block of images consists of 32 images distributed over six flight lines four of them in the east - west direction and two in north south direction the IOP are known through careful camera calibration procedure, also 37 ground control points are known through terrestrial surveying procedure. The exact location of camera station at time of exposure is known through GPS/INS integration process (EOP).

The configuration of flight lines, camera station and ground control points is illustrated in the following figure.

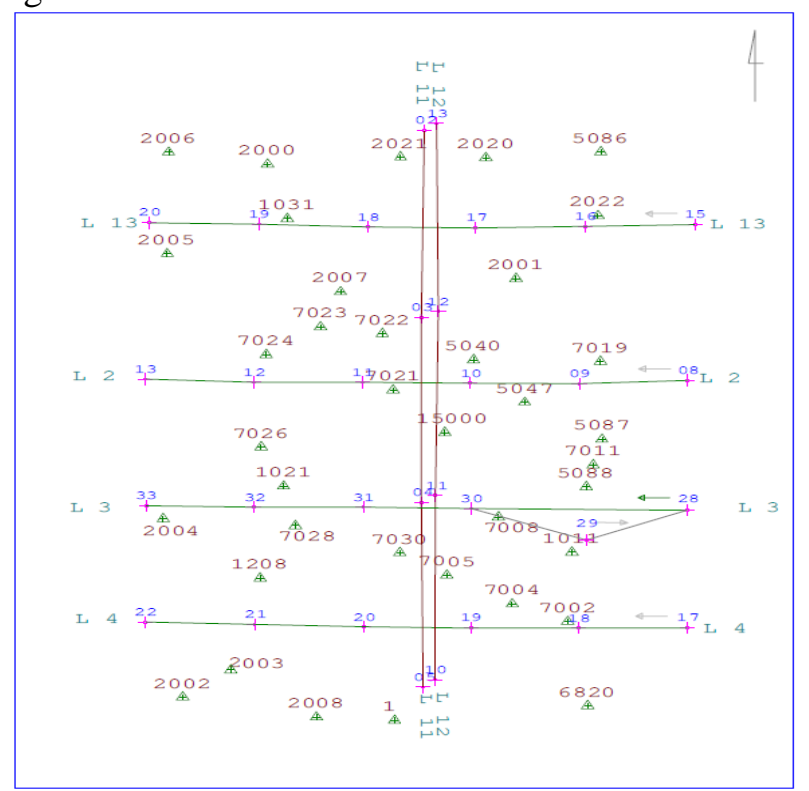

Figure 4. Data set configuration

In order to analysis and compare the two technologies of Direct Georeferencing and GPSsupported AT we will take the $37 \mathrm{GCP}$ as a reference points. Then dealing with each pair of photo and using the image coordinates measurements for GC points, EOP (from GPS/INS integration) and IOP (from camera calibration) to determine $\lambda$ and $\mu$ (scale factor) for each Ground control point through least squares.

$$
\left[\begin{array}{c}
X_{o_{r}}-X_{o_{l}} \\
Y_{o_{r}}-Y_{o_{l}} \\
Z_{o_{r}}-Z_{o_{l}}
\end{array}\right]=\emptyset R_{\left(\omega_{l}, \hat{l}_{1}, \kappa_{l}\right)}\left[\begin{array}{c}
x_{l}-x_{p} \\
y_{l}-y_{p} \\
-c
\end{array}\right]-\left(\mu R_{\left(\omega_{r}, \hat{o}_{r}, \kappa_{r}\right)}\left[\begin{array}{c}
x_{r}-x_{p} \\
y_{r}-y_{p} \\
-c
\end{array}\right]\right. \text { (2) }
$$

Then taking every pair of photos and intersecting the measured GCP to the object space to come up with its ground coordinates using a MATLAB code.

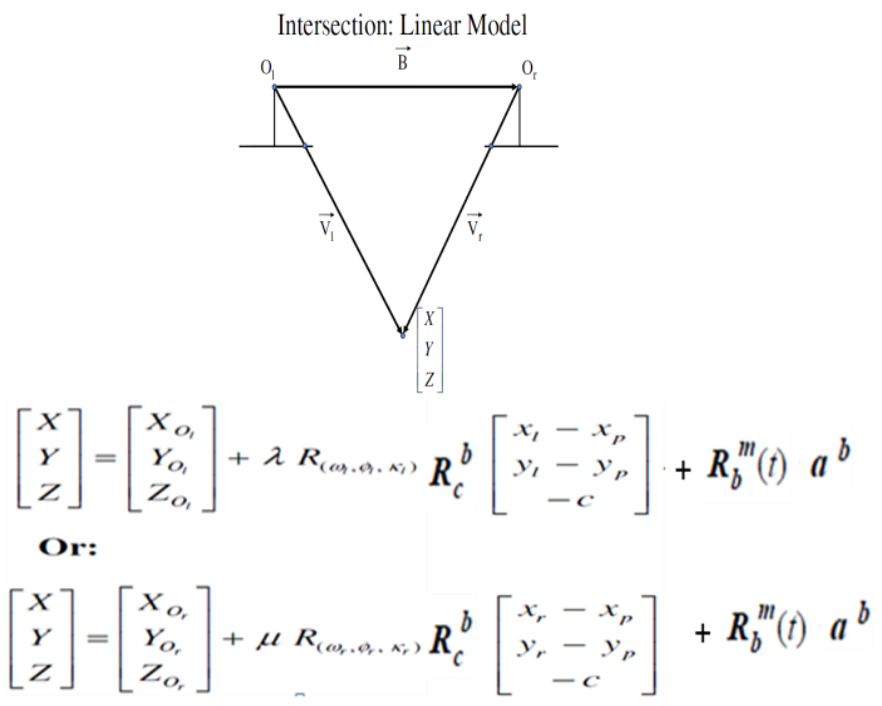

Figure 5. Space intersection Model

This procedure was repeated while the following errors were introduced to the EOP parameters:

- $10 \mathrm{~cm}$ in Xo, Yo and Zo

- 10 arc sec in $\omega$ and $\varphi$ attitude angles.

- $10 \mathrm{~cm}$ in Xo ,Yo and Zo and 10 arc sec in $\omega$ and $\varphi$ attitude angles.

- $20 \mathrm{~cm}$ in Xo ,Yo and Zo and 20 arc sec in $\omega$ and $\varphi$ attitude angles.

- 5 arc min. in $\omega$ and $\varphi$ attitude angles.

Then similar error was introduced in the Boresight elements (Boresight angles and lever arm components)

- $10 \mathrm{~cm}$ in X,Y and Z Lever arm component.

- 10 arc sec in $\omega$ and $\varphi$ Boresight angles.

- $10 \mathrm{~cm}$ in $\mathrm{X}, \mathrm{Y}$ and $\mathrm{Z}$ Lever arm component and 10 arc sec in $\omega$ and $\varphi$ Boresight angles.

- $20 \mathrm{~cm}$ in $\mathrm{X}, \mathrm{Y}$ and $\mathrm{Z}$ Lever arm component and 20 arc sec in $\omega$ and $\varphi$ Boresight angles.

- 5 arc min. in $\omega$ and $\varphi$ attitude angles.

These results were then compared with two sets coming from the ISO one with no GCP taken and the other with 1 FULL GCP applying the same configuration and biases using the MSAT software. For the evaluation of the accuracy potential of direct Georeferencing we used the software MSAT (Multi Sensor Aerial Triangulation) developed by Digital Photogrammetry Research Group at the department of Geomatics, University of Calgary. 


\section{PERFORMANCE CRITERION}

The performance of these different scenarios is evaluated through Root Mean Square Error Analysis:-

- Compares the adjusted ground coordinates from the ISO or Direct Georeferencing procedures with the true values of the GCPs used.

- This criterion is very important since it addresses the quality of the reconstructed object space (the ultimate objective of photogrammetric mapping).

5. RESULTS AND ANALYSIS

For the first group of errors (EOP parameters)

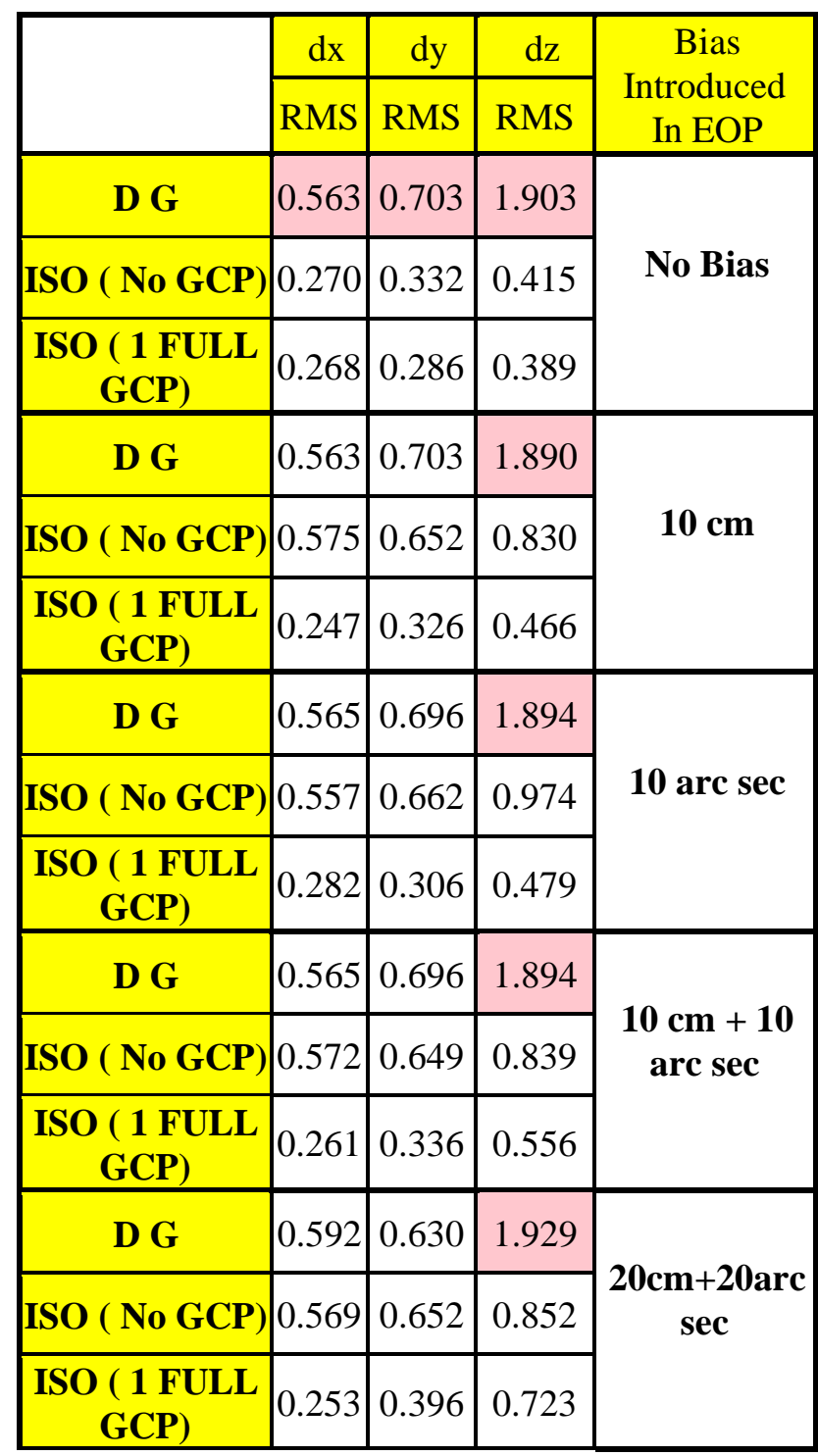

\begin{tabular}{|c|c|c|c|c|}
\hline D G & 0.720 & 1.076 & 2.980 & \multirow{2}{*}{5 min. } \\
\cline { 1 - 3 } ISO ( No GCP) & 0.509 & 0.663 & 2.285 & \\
\cline { 1 - 3 } $\begin{array}{c}\text { ISO ( 1 FULL } \\
\text { GCP) }\end{array}$ & 0.547 & 0.730 & 2.494 & \\
\hline
\end{tabular}

Table 1. Errors introduced in EOP

For the second group of error (Boresight angles and lever arm components)

\begin{tabular}{|c|c|c|c|c|}
\hline & $d x$ & dy & dz & \multirow{2}{*}{$\begin{array}{c}\text { Bias In } \\
\text { Boresight } \\
\text { and Lever } \\
\text { Arm } \\
\end{array}$} \\
\hline & RMS & RMS & RMS & \\
\hline D G & 0.517 & 0.701 & 1.894 & \multirow{3}{*}{$10 \mathrm{~cm}$} \\
\hline $\begin{array}{c}\text { ISO ( No } \\
\text { GCP) }\end{array}$ & 0.570 & 0.650 & 0.834 & \\
\hline $\begin{array}{l}\text { ISO ( } 1 \text { FULL } \\
\text { GCP) }\end{array}$ & 0.521 & 0.561 & 0.830 & \\
\hline D G & 0.536 & 0.565 & 1.912 & \multirow{3}{*}{10 arc sec } \\
\hline $\begin{array}{c}\text { ISO ( No } \\
\text { GCP) }\end{array}$ & 0.576 & 0.664 & 0.840 & \\
\hline $\begin{array}{c}\text { ISO ( } 1 \text { FULL } \\
\text { GCP) }\end{array}$ & 0.534 & 0.576 & 0.820 & \\
\hline D G & 0.532 & 0.564 & 1.915 & \multirow{3}{*}{$\begin{array}{c}10 \mathrm{~cm}+10 \\
\operatorname{arc~sec}\end{array}$} \\
\hline $\begin{array}{c}\text { ISO ( No } \\
\text { GCP) }\end{array}$ & 0.572 & 0.652 & 0.845 & \\
\hline $\begin{array}{c}\text { ISO ( } 1 \text { FULL } \\
\text { GCP) }\end{array}$ & 0.520 & 0.557 & 0.837 & \\
\hline D G & 1.194 & 0.510 & 1.945 & \multirow{3}{*}{$\begin{array}{c}20 \mathrm{~cm}+20 \mathrm{arc} \\
\mathrm{sec}\end{array}$} \\
\hline $\begin{array}{c}\text { ISO ( No } \\
\text { GCP) }\end{array}$ & 0.570 & 0.662 & 0.867 & \\
\hline $\begin{array}{c}\text { ISO ( } 1 \text { FULL } \\
\text { GCP) } \\
\end{array}$ & 0.506 & 0.546 & 0.865 & \\
\hline D G & 1.368 & 1.125 & 4.564 & \multirow{3}{*}{$5 \mathrm{~min}}$. \\
\hline $\begin{array}{c}\text { ISO ( No } \\
\text { GCP) }\end{array}$ & 0.702 & 1.053 & 2.811 & \\
\hline $\begin{array}{c}\text { ISO ( } 1 \text { FULL } \\
\text { GCP) }\end{array}$ & 0.486 & 0.582 & 2.508 & \\
\hline
\end{tabular}

Table 2. Errors introduced in Boresight 


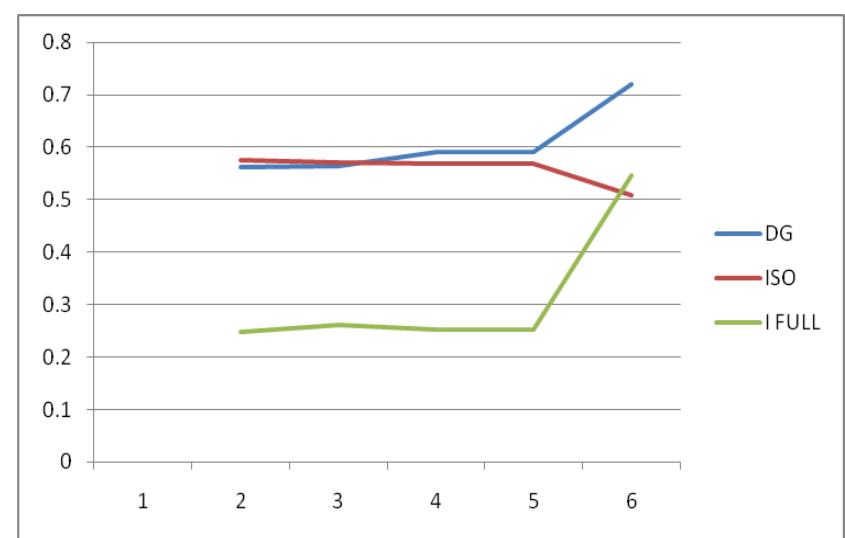

Figure 6 overall behavior of $\mathrm{dX}$ with different biases applied in EOP

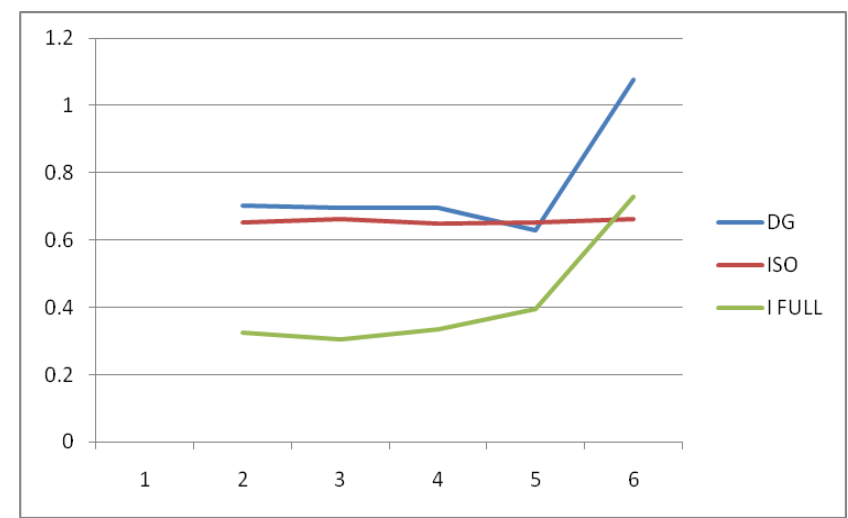

Figure 7. overall behavior of $\mathrm{dY}$ with different biases applied in EOP

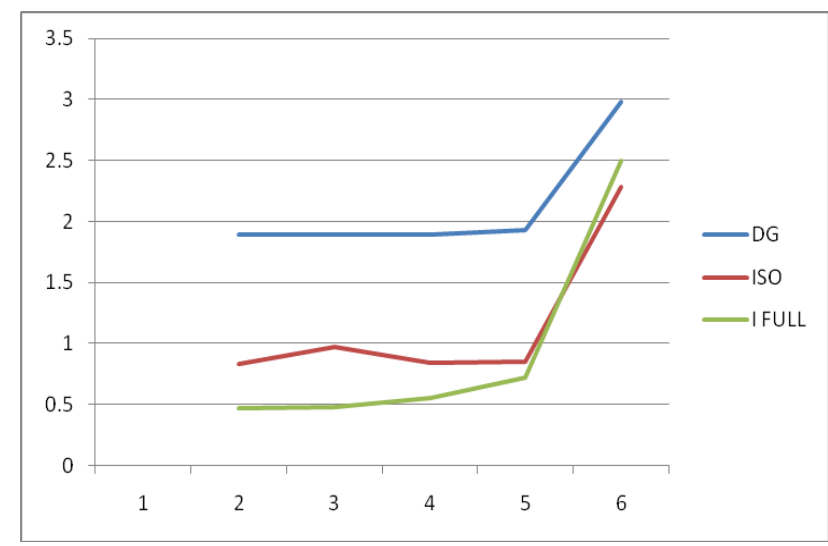

Figure 8. overall behavior of $\mathrm{dZ}$ with different biases applied in EOP

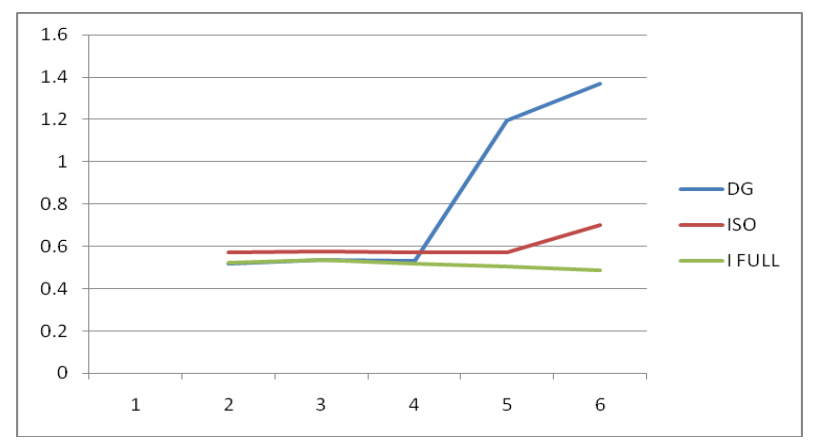

Figure 9. overall behavior of $\mathrm{dX}$ with different biases applied in Boresight

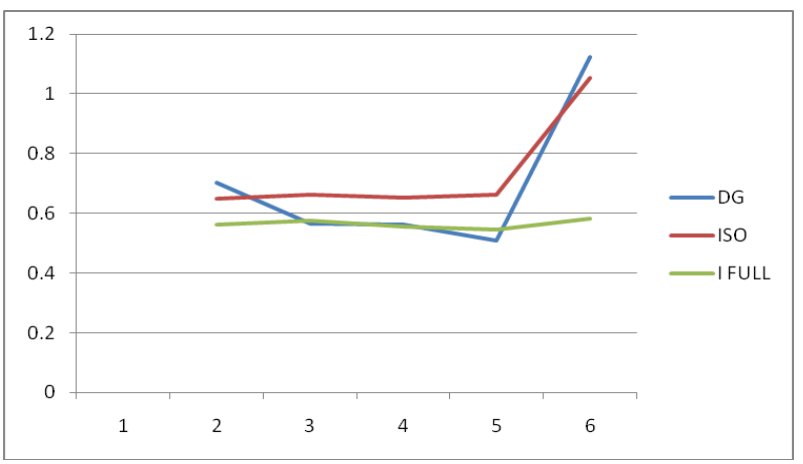

Figure10. overall behavior of dY with different biases applied in Boresight

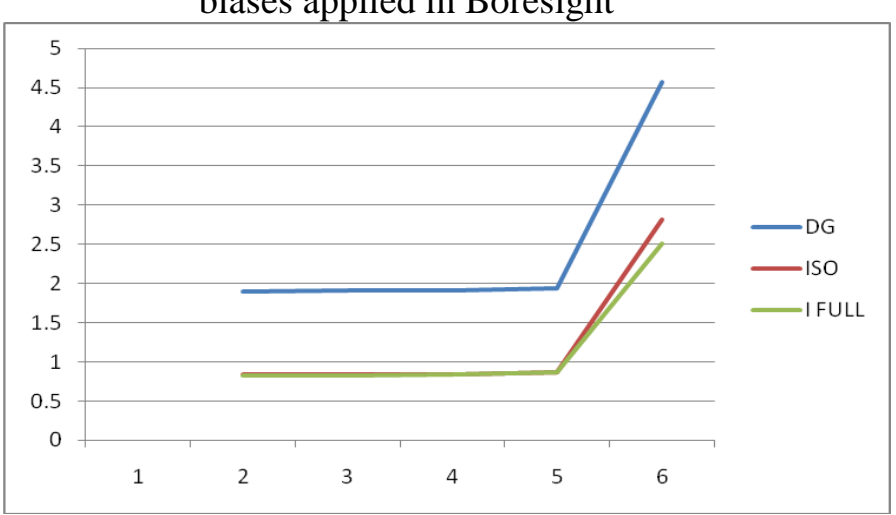

Figure 11. overall behavior of $\mathrm{dZ}$ with different biases applied in Boresight that:

From the previous tables and figures we can say

- Using ISO helps in improving the vertical accuracy in general.

- Using GCP in ISO almost yields equivalent horizontal and vertical accuracy in the case of no Bias introduced.

- While using 1 FULL GCP significantly improves the accuracy of horizontal and vertical components - in case of biases is introduced and kept it to its minimum values.

- Generally ISO significantly improves the point precision if either no biases or a bias is introduced in EOP components.

- The importance of system calibration in determining the Boresight angles and lever arm components as its equal effect in the point position accuracy comparing to the importance of the quality of the EOP.

- In case of small errors introduced $(10 \mathrm{~cm}, 10$ arc sec) we will find convergence in error values in the $\mathrm{X}$ and $\mathrm{Y}$ component between DG and ISO (without GCP), while in the case of large error introduced ( $5 \mathrm{~min}$. ) the DG will diverge away 
from ISO, where the error in $\mathrm{Z}$ component will be better in all cases of biases with the ISO 1 Full GCP.

- In the absence of Biases, direct and indirect orientation yield comparable results.

- In the presence of Biases, indirect orientation produces better results than direct orientation.

\section{CONCLUSIONS}

- In the DG Correcting overall system calibration including the GPS/inertial component as well as the imaging sensor itself is the limiting factor to achieve good object space.

- GPS-supported AT has already helped to improve the performance of the conventional aerial triangulation process.

- Directly derived exterior orientation parameters using combined GPS and highperformance INS systems offer the possibility of eliminating conventional aerial triangulation in the long run.

- The result of this test indicated that the DG and GPS-supported AT have similar accuracy. Comparing with the conventional aerial photography method, the two technologies reduced the dependence on ground control (used only for quality control purposes).

- From the workload of data disposal, DG has much obvious advantage than conventional AT technique.

\section{REFERENCES}

Ackermann, F. (1995). Sensor-and-Data Integration. The New Challenge, Integrated Sensor Orientation. . C. Navaro: 2-10.

El-Sheimy, N. (2008). Georeferencing Component of LiDAR Systems. Topographic Laser Ranging and Scanning: Principles and Processing

El-sheimy, $\quad N$. (2008). Land mobile mapping system. Advances in Photogrammetry, Remote Sensing and Spatial Information Sciences. J. C. Zhilin Li and E. Baltsavias, Taylor \& Francis group.

Habib, A. F., M. S. Ghanma, et al. (2005). "Image georeferencing using LIDAR data." IGARSS 2005: IEEE International Geoscience and
Remote Sensing Symposium, Vols 1-8, Proceedings: 1158-1161.

Schwarz, K. P. and N. El-Sheimy (1996). Kinematic Multi-sensor Systems For Close Range Digital Mapping. ISPRS Commission V, Working Group III,, Vienna, Austria. 